\title{
Reoperation rates and impact on outcome in a large, prospective, multicenter, adult spinal deformity database
}

\author{
Clinical article
}

\author{
Justin K. Scheer, B.S., ${ }^{1}$ Jessica A. Tang, B.S., ${ }^{1}$ Justin S. Smith, M.D., Ph.D., ${ }^{2}$ Eric KLineberg, M.D., ${ }^{3}$ \\ Robert A. Hart, M.D., ${ }^{4}$ Gregory M. Mundis JR., M.D.,${ }^{5}$ Douglas C. Burton, M.D., ${ }^{6}$ \\ Richard Hostin, M.D. ${ }^{7}$ Michael F. O’Brien, M.D. ${ }^{7}$ Shay Bess, M.D..${ }^{8}$ Khaled M. Kebaish, M.D., 9 \\ Vedat Deviren, M.D., ${ }^{10}$ Virginie Lafage, Ph.D., ${ }^{11}$ Frank Schwab, M.D., 11 \\ Christopher I. Shaffrey, M.D., ${ }^{2}$ Christopher P. Ames, M.D., ${ }^{12}$ and the International Sine Study Group
}

${ }^{1}$ University of California, San Diego, School of Medicine; ${ }^{5}$ San Diego Center for Spinal Disorders, La Jolla; ${ }^{3}$ Department of Orthopaedic Surgery, University of California, Davis; Departments of ${ }^{10}$ Orthopedic Surgery and ${ }^{12}$ Neurological Surgery, University of California, San Francisco, California; ${ }^{2}$ Department of Neurosurgery, University of Virginia Health System, Charlottesville, Virginia; ${ }^{4}$ Department of Orthopaedic Surgery, Oregon Health \& Science University, Portland, Oregon; ${ }^{6}$ Department of Orthopedic Surgery, University of Kansas Medical Center, Kansas City, Kansas; ${ }^{7}$ Department of Orthopedic Surgery, Baylor Scoliosis Center, Plano, Texas; ${ }^{8}$ Rocky Mountain Hospital for Children, Denver, Colorado; ${ }^{9}$ Department of Orthopaedic Surgery, Johns Hopkins University, Baltimore, Maryland; and "Department of Orthopaedic Surgery, NYU Hospital for Joint Diseases, New York, New York

Object. Complications and reoperation for surgery to correct adult spinal deformity are not infrequent, and many studies have analyzed the rates and factors that influence the likelihood of reoperation. However, there is a need for more comprehensive analyses of reoperation in adult spinal deformity surgery from a global standpoint, particularly focusing on the 1st year following operation and considering radiographic parameters and the effects of reoperation on health-related quality of life (HRQOL). This study attempts to determine the prevalence of reoperation following surgery for adult spinal deformity, assess the indications for these reoperations, evaluate for a relation between specific radiographic parameters and the need for reoperation, and determine the potential impact of reoperation on HRQOL measures.

Methods. A retrospective review was conducted of a prospective, multicenter, adult spinal deformity database collected through the International Spine Study Group. Data collected included age, body mass index, sex, date of surgery, information regarding complications, reoperation dates, length of stay, and operation time. The radiographic parameters assessed were total number of levels instrumented, total number of interbody fusions, C-7 sagittal vertical axis, uppermost instrumented vertebra (UIV) location, and presence of 3-column osteotomies. The HRQOL assessment included Oswestry Disability Index (ODI), 36-Item Short Form Health Survey physical component and mental component summary, and SRS-22 scores. Smoking history, Charlson Comorbidity Index scores, and American Society of Anesthesiologists Physical Status classification grades were also collected and assessed for correlation with risk of early reoperation. Various statistical tests were performed for evaluation of specific factors listed above, and the level of significance was set at $\mathrm{p}<0.05$.

Results. Fifty-nine (17\%) of a total of 352 patients required reoperation. Forty-four $(12.5 \%)$ of the reoperations occurred within 1 year after the initial surgery, including 17 reoperations (5\%) within 30 days.

Two hundred sixty-eight patients had a minimum of 1 year of follow-up. Fifty-three (20\%) of these patients had a 3-column osteotomy, and $10(19 \%)$ of these 53 required reoperation within 1 year of the initial procedure. However, 3-column osteotomy was not predictive of reoperation within 1 year, $\mathrm{p}=0.5476$ ). There were no significant differences between groups with regard to the distribution of UIV, and UIV did not have a significant effect on reoperation rates. Patients needing reoperation within 1 year had worse ODI and SRS-22 scores measured at 1-year follow-up than patients not requiring operation.

Conclusions. Analysis of data from a large multicenter adult spinal deformity database shows an overall $17 \%$ reoperation rate, with a $19 \%$ reoperation rate for patients treated with 3-column osteotomy and a $16 \%$ reoperation rate for patients not treated with 3-column osteotomy. The most common indications for reoperation included instrumentation complications and radiographic failure. Reoperation significantly affected HRQOL outcomes at 1-year follow-up. The need for reoperation may be minimized by carefully considering spinal alignment, termination of fixation, and type of surgical procedure (presence of osteotomy). Precautions should be taken to avoid malposition or instrumentation (rod) failure.

(http://thejns.org/doi/abs/10.3171/2013.7.SPINE12901)

\section{KEY WoRdS • spinal deformity • reoperation • HRQOL • quality of life}

Abbreviations used in this paper: ASA = American Society of Anesthesiologists performance status; BMI = body mass index; HRQOL $=$ health-related quality of life; ISSG $=$ International Spine Study Group; ODI = Oswestry Disability Index; SF-36 = 36-Item Short Form Health Survey; SRS-22 = Scoliosis Research Society 22-item patient questionnaire; SVA = sagittal vertical axis; UIV = uppermost instrumented vertebra.

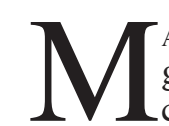

ANAGEMENT of adult spinal deformity poses great challenges to the surgeon and has historically been associated with relatively high rates

This article contains some figures that are displayed in color online but in black-and-white in the print edition. 
of complications and the need for reoperation. ${ }^{20}$ Studies have documented the complications that arise, assessing the rates of reoperation, analyzing factors that increase the likelihood of reoperation, and suggesting methods to prevent the need for reoperation. These studies not only reported complications following primary spine surgery $y^{3,8}$ but also compared complication rates between different types of spine surgeries ${ }^{14,15}$ and between primary and revision procedures. ${ }^{6,11,12}$ Rates of reoperation are frequently cited, ranging from $10 \%$ to $25 \%$, and among the many reasons behind reoperation-including infection, curve progression, proximal junction kyphosis, implant failure, and removal of painful implants ${ }^{4,13,17}$ - pseudarthrosis emerges as one of the most commonly documented indications. ${ }^{6,9,10,13,16-18}$ There are also studies that have investigated the effects of demographic factors, suggesting older age as a risk factor for developing pseudarthrosis, with the consequent need for revision surgery. ${ }^{1,5,7}$ However, few studies ${ }^{9,10,19}$ have focused on the effects of reoperation and pseudarthrosis on patients' scores on outcome measures, including the SRS-22 and the ODI. While most of these studies address in detail the challenges of spine surgery that frequently result in reoperation, each examines a collection of individual issues, such as specific complications, risk factors, techniques to prevent reoperation, or outcome measures. However, there has yet to be a comprehensive analysis combining all aspects related to reoperation in adult spinal deformity surgery from a global perspective, with a focus on the relatively shortterm setting of the 1st year following surgery.

Furthermore, there is a need to investigate the factors that influence rates of complications and reoperations across key time frames that are currently used as quality measures by governmental agencies and third-party payers. Reoperation within 30 days and reoperation within 1 year may be important quality metrics that will require baseline rates at centers of excellence to set acceptable occurrence standards for these events. HRQOL measures can also be used to compare the rate of the recovery and clinical change timeline of reoperation versus primary surgery to determine the extent to which a reoperation may slow or potentially permanently limit clinical improvement following deformity surgery.

This study attempts to address in detail the following 4 primary aims: 1) determine prevalence of reoperation following surgery for adult spinal deformity, 2) assess indications for these reoperations, 3) evaluate for a relation between specific radiographic parameters and the need for reoperation, and 4) determine the potential impact of reoperation on HRQOL measures.

\section{Methods}

This study is a retrospective review of a prospective, multicenter adult thoracolumbar spinal deformity database of the International Spine Study Group (ISSG), which is composed of 11 sites across the United States. Data from consecutive cases involving patients treated between 2010 and 2012 were obtained, and all patients were enrolled into an institutional review board-approved protocol by the respective sites. Inclusion criteria for the ISSG database are: age of at least 18 years and presence of spinal deformity, as defined by a Cobb angle of at least $20^{\circ}$; an SVA measurement (distance between the C-7 plumb line and posterior superior margin of the sacrum) of at least $5 \mathrm{~cm}$; a pelvic tilt of at least $25^{\circ}$; and/ or thoracic kyphosis of at least $60^{\circ}$. Exclusion criteria included spinal deformity of a neuromuscular etiology and the presence of active infection or malignancy.

Data collected included age, sex, date of surgery, complications, reoperation dates, and HRQOL outcomes. Reoperation was defined as any unplanned return to the operating room as a result of the original surgery. The reoperation indications were divided into the following categories: instrumentation malposition/rod fracture, radiographic (proximal junction failure, distal junction failure, pseudarthrosis, coronal malalignment) neurological compromise, infection, medical (cardiopulmonary, vascular gastrointestinal, renal), operative, and wound. The instrumentation malposition/fracture category described situations in which there was implant failure or migration, malpositioning, painful implants, or bony fracture due to implants not related to proximal junction failure.

Other variables that were assessed included BMI, the total number of anterior and posterior levels instrumented, the total number of interbody fusions, preoperative SVA measurement, total operative time (minutes), length of hospital stay (days), the uppermost instrumented vertebra (UIV) location (characterized as upper thoracic for fixation with superior termination between T-2 and T-8 and inferior fixation between L-5 and the ilium, thoracolumbar for fixation with superior termination between T-8 and L-3 and inferior fixation between L-4 and the ilium, middle for fixation with superior termination between T-1 and T- 8 to and inferior fixation between T-12 and L-5, and lumbar for fixation with superior fixation between L-1 and L-5 and inferior fixation between L-4 and the ilium), and the presence of 3-column osteotomies (including pedicle subtraction osteotomy and vertebral column resection). Smoking history, Charlson Comorbidity Index scores, and American Society of Anesthesiologists performance status grades (ASA grades) were also collected and assessed for correlation with risk of early reoperation.

The HRQOL measures were collected preoperatively and at 1 and 2 years after the index surgery and included ODI, SF-36 mental and physical component summary, back and leg pain numerical rating scale (range 0 [no pain] to 10 [maximal pain]), and SRS-22. ${ }^{2}$ Analyses of HRQOL in the present study were confined to patients who had a minimum of 1 year of follow-up of at least one standardized measure. A subset analysis was performed using data from only patients with a minimum of 2 years of follow-up.

Patients were divided between 2 groups based on whether a reoperation was performed. Patients who underwent repeat surgery were further grouped into the following 4 categories based on the time from the date of index surgery: less than 30 days (the Reop Within 30 Days Group), between 30 days and 1 year (the Reop 30 Days-1 Year Group), less than 1 year (the Reop Within 1 Year Group, which includes patients in the "within 30 
days" category), and more than 1 year (the Reop After 1 Year Group).

Statistical analysis was performed using the Student t-test for normally distributed data and the Wilcoxon rank-sum or Kruskal-Wallis tests when appropriate for non-normal data distributions. All statistical analyses were conducted using commercially available software (JMP v5.0, SAS Institute, Inc.), and the level of significance was set at $\mathrm{p}<0.05$.

\section{Results}

\section{Incidence of Reoperation}

The prospective multicenter database included a total of 352 patients enrolled between October 2010 and October 2012. The overall mean follow-up was 1.6 years (range 6 weeks to 2 years), and during this follow-up interval, a total of $59(17 \%)$ of 352 patients required reoperation. In 44 cases $(75 \%$ of the reoperation group and $12.5 \%$ of all cases in the database), these reoperations occurred within 1 year following initial surgery, including 17 (5\%) within 30 days of initial surgery. Seven patients required a minimum of 2 reoperations, and of those 7 patients, 4 required 3 reoperations. Of the total 352 patients in the database, 268 patients $(76 \%)$ had a minimum of 1 year of follow-up, and $169(48 \%)$ had 2 years of follow-up. Of the patients with at least 1 year of follow-up $(n=268), 46(17 \%)$ required reoperation at some point, with $31(12 \%)$ of these procedures being within 1 year of the index surgery and $12(4 \%)$ being within 30 days of the index surgery.

\section{Indications for Reoperation}

Reoperation indications for all patients requiring reoperation $(n=59)$ included the following: instrumentation malposition/fracture $(n=19)$, radiographic $(n=19)$, infection $(n=7)$, neurological compromise $(n=6)$, operative $(n=2)$, wound $(n=2)$, medical $(n=1)$, both neurological and radiographic $(n=2)$, and neurological, operative, and wound combined $(n=1)$. For those patients who underwent reoperation within 30 days of the index surgery $(\mathrm{n}=$ 17; subset of the total of 59 who required reoperation), the most prominent reason was neurological compromise (n $=4)$. Other indications were described as instrumentation malposition/fracture $(n=3)$, infection $(n=3)$, radiographic $(n=2)$, wound $(n=2)$, medical $(n=1)$, both neurological and operative $(n=1)$, and neurological, operative, and wound combined $(\mathrm{n}=1)$.

\section{Demographic and Surgical Results}

All results represent only the cohort of patients with a minimum of 1 year of follow-up $(n=268)$, and the averages with standard deviations are presented in Table 1. There were no significant differences in age between patients requiring reoperation within 1 year of the index surgery (mean age $56 \pm 15$ years, $n=31$ [including the 12 patients who required reoperation within 30 days]), within 30 days of the index surgery $(47 \pm 19$ years, $n=$ $12)$, more than 1 year after the index surgery $(53 \pm 16$ years, $n=15)$, and not requiring any reoperation $(55 \pm 15$ years, $\mathrm{n}=222$, $\mathrm{p}>0.05$ for all comparisons). However, patients who underwent reoperation within 30 days of index surgery were significantly younger (mean age $47 \pm 19$ years) than patients who underwent reoperation between 30 days and 1 year after the index procedures $(60 \pm 13$ years, $\mathrm{p}=0.02808$ ).

There were no significant differences between the groups of patients who did not undergo reoperation or underwent reoperation within 30 days, between 30 days and 1 year, within 1 year, or more than 1 year of index surgery for the rest of the variables including preoperative BMI, Charlson comorbidity index, ASA grade, or smoking history $(p>0.05)$. Patients who underwent reoperation within 30 days of the index procedure had a significantly smaller preoperative SVA $(2.0 \pm 5.7)$ than patients who underwent reoperation between 30 days and 1 year after the index procedure $(7.0 \pm 6.3, \mathrm{p}=0.0219)$, but there were no other significant differences between the groups with respect to preoperative SVA measurement, number of anterior or posterior instrumented levels, total number of interbody fusions, total operative time, and length of hospital stay $(\mathrm{p}>0.05)$.

Of the total patient group $(\mathrm{n}=268), 53$ patients $(20 \%)$ had a 3 -column osteotomy and 10 (19\%) of the 53 required reoperation. Six of these reoperations occurred within 1 year of the index procedure, and 4 occurred more than 1 year after the index procedure. However, 3-column osteotomy was not predictive of reoperation at any time point $(\mathrm{p}=0.5476)$. Three patients required a second reoperation during the study period, and 2 of these patients had been treated with a 3-column osteotomy as part of the initial procedure. The indications for reoperation for patients who had been treated with 3-column osteotomies were implant failure $(n=4)$, neurological compromise ( $=3)$, radiographic $(n=2)$, and operative $(n=1)$.

In the 46 patients who underwent reoperation (out of 268 patients), the UIV was classified as follows: upper thoracic in 15 cases (33\%), thoracolumbar in 25 cases $(54 \%)$, middle in 5 cases (11\%), and lumbar in 1 case $(2 \%)$. For those patients who underwent reoperation within 1 year $(\mathrm{n}=31)$, the UIV was classified as upper thoracic in 10 cases $(5 \%)$, thoracolumbar in 16 cases $(10 \%)$, middle in 4 cases $(2 \%)$, and lumbar in 1 case $(1 \%)$. For the 222 patients who did not undergo reoperation, the UIV was classified as upper thoracic in 70 cases (32\%), as thoracolumbar in 95 cases (43\%), as middle in 42 cases (19\%), as lumbar in 6 cases (3\%), and as unknown in 9 cases (4\%). There were no significant differences between groups with regard to the distribution of UIV, and UIV did not have a significant effect on reoperation rates

\section{Reoperation Impact on Clinical Outcome}

Reoperation Within 1 Year Versus No Reoperation. There were no significant preoperative differences for HRQOL measures $(p>0.05)$. At 1-year follow-up, the Reop Within 1 Year Group had a significantly higher mean leg pain score $(3.8 \pm 3.2)$ than the patients who had not undergone reoperation (the No Reop Group, $3.3 \pm$ $2.9, \mathrm{p}=0.0026)$. There was no significant difference in back pain scores between the 2 groups $(p>0.05)$. (Note: All follow-up times in this paper refer to the time since 
Reoperation rates and impact on outcome

TABLE 1: Demographic and surgical data for patients with at least 1 year of follow-up*

\begin{tabular}{lccccccc}
\hline Parameter at Index Surgery & $\begin{array}{c}\text { All Pts } \\
(\mathrm{n}=268)\end{array}$ & $\begin{array}{c}\text { No Reop } \\
(\mathrm{n}=222)\end{array}$ & $\begin{array}{c}\text { All Reop } \\
(\mathrm{n}=46)\end{array}$ & $\begin{array}{c}\text { Reop After 1 } \\
\operatorname{Yr}(\mathrm{n}=15)\end{array}$ & $\begin{array}{c}\text { Reop w/in 1 Yr } \\
(\mathrm{n}=31)\end{array}$ & $\begin{array}{c}\text { Reop w/in 30 } \\
\text { Days }(\mathrm{n}=12)\end{array}$ & $\begin{array}{c}\text { Reop 30 Days-1 } \\
\text { Yr }(\mathrm{n}=19)\end{array}$ \\
\hline age (yrs) & $55 \pm 15$ & $55 \pm 15$ & $55 \pm 15$ & $53 \pm 16$ & $56 \pm 15$ & $47 \pm 19$ & $62 \pm 9$ \\
male/female & $38: 231$ & $30: 192$ & $8: 38$ & $2: 13$ & $6: 25$ & $2: 10$ & $4: 15$ \\
BMI & $27.2 \pm 6.5$ & $27.3 \pm 6.7$ & $26.7 \pm 5.6$ & $26.8 \pm 6.9$ & $26.7 \pm 5.0$ & $27.6 \pm 5.7$ & $26.2 \pm 4.6$ \\
smoking & 23 & 18 & 5 & 0 & 2 & 2 & 2 \\
ASA & $2.3 \pm 0.7$ & $2.2 \pm 0.7$ & $2.3 \pm 0.7$ & $2.3 \pm 0.6$ & $2.3 \pm 0.7$ & $2.1 \pm 0.7$ & $2.4 \pm 0.8$ \\
CCl & $1.4 \pm 1.6$ & $1.4 \pm 1.6$ & $1.6 \pm 1.7$ & $2.1 \pm 2.1$ & $1.3 \pm 1.4$ & $1.1 \pm 1.1$ & $1.4 \pm 1.5$ \\
preop SVA (cm) & $5.3 \pm 7.6$ & $5.1 \pm 7.4$ & $5.7 \pm 8.3$ & $6.9 \pm 11.8$ & $5.2 \pm 6.6$ & $2.0 \pm 5.7$ & $7.3 \pm 6.3$ \\
anterior instrumented levels & $4.3 \pm 3.9$ & $3.9 \pm 3.2$ & $5.6 \pm 6.2$ & $3.7 \pm 0.6$ & $6.4 \pm 7.4$ & $4.3 \pm 0.5$ & $9.3 \pm 11.8$ \\
posterior instrumented levels & $11.5 \pm 4.8$ & $11.6 \pm 4.8$ & $11.6 \pm 4.5$ & $11.3 \pm 4.3$ & $11.7 \pm 4.7$ & $12.6 \pm 3.9$ & $11.1 \pm 5.2$ \\
interbody fusions & $2.6 \pm 1.9$ & $2.5 \pm 1.8$ & $3.0 \pm 2.0$ & $3.3 \pm 2.3$ & $2.9 \pm 1.9$ & $3.2 \pm 2.7$ & $2.8 \pm 1.4$ \\
presence of 3CO (\% of group) & $53(20.0 \%)$ & $43(19.4 \%)$ & $10(21.7 \%)$ & $4(26.7 \%)$ & $6(19.4 \%)$ & $3(25.0 \%)$ & $3(15.8 \%)$ \\
operative time (minutes) & $391 \pm 131$ & $387 \pm 127$ & $410 \pm 149$ & $403 \pm 146$ & $413 \pm 153$ & $422 \pm 147$ & $407 \pm 158$ \\
length of stay (days) & $8.7 \pm 10.5$ & $8.1 \pm 4.9$ & $11.3 \pm 22.5$ & $7.6 \pm 4.1$ & $13.0 \pm 27.0$ & $7.2 \pm 1.8$ & $8.9 \pm 4.6$ \\
\hline
\end{tabular}

* Stratification of subgroups by time is based on time from the index surgery to reoperation. Values are shown as means with SDs or numbers of patients (\% of group). $\mathrm{CCl}=$ Charlson Comorbidity Index; pts = patients; reop = reoperation; $3 \mathrm{CO}=3$-column osteotomy.

the initial surgery.) The ODI was significantly higher at 1-year follow-up for the Reop Within 1 Year group (34.2 $\pm 21.2)$ than the No Reop Group $(25.7 \pm 19.2, p=0.0411$, Fig. 1). With respect to 1-year SRS-22 scores, the Reop Within 1 Year Group had significantly lower scores than the No Reop Group for the activity $(3.2 \pm 1.0$ vs $3.6 \pm$ $0.9, \mathrm{p}=0.0418)$, appearance $(3.3 \pm 1.1$ vs $3.8 \pm 0.8, \mathrm{p}=$ $0.0286)$, and pain $(31 . \pm 1.1$ vs $3.5 \pm 1.0, \mathrm{p}=0.0469)$ subcategories as well as significantly lower total scores (3.4 \pm 0.9 vs $3.70 .8, p=0.0351$; Fig. 2$)$. There was no statistically significant difference between the 2 groups with respect to the mental or satisfaction subcategory scores ( $p>$ 0.05). Furthermore, the Reop Within 1 Year Group had a significantly smaller change in SF-36 physical component summary scores (difference between baseline and 1-year score) and a smaller change in SRS total scores than the No Reop Group (respectively, $1.5 \pm 11.9$ vs. $7.9 \pm 10.9, \mathrm{p}=$ 0.0228 ; and $0.5 \pm 0.7$ vs. $0.9 \pm 0.7, p=0.0070$ ). There were no significant differences in any of the HRQOL outcomes between the 2 groups at 2 years after the index surgery ( $p$ $>0.05)$.

Reoperation After 1 Year Versus No Reoperation. There were no significant differences with respect to any of the HRQOL outcomes at 1- or 2-year time points between the Reop After 1 Year Group and the No Reop Group ( $p>0.05)$ with one exception. The No Reop Group had a larger 2-year change in SF-36 physical component summary scores (compared with preoperative values) than the Reop After 1 Year Group $(8.7 \pm 10.9$ vs $1.8 \pm 8.9$, $\mathrm{p}=0.0251$ ).

Reoperation Within 30 Days Versus 30 Days-1 Year After the Index Surgery. Patients in the Reop Within 30 Days Group had significantly lower preoperative ODI scores (mean $33.6 \pm 19.3$ ) than those in the Reop 30 Days-1 Year Group (mean $47.9 \pm 17.1, \mathrm{p}=0.0387$ ). The only significant difference between the groups at 1 year after the index surgery was found to be in SRS pain scores, with the Reop Within 30 Days Group having a higher score $(3.6 \pm 1.0$ vs $2.8 \pm 1.0, \mathrm{p}=0.0472)$. At 2 years after the index surgery, patients in the Reop Within 30 Days Group had significantly better mean SRS-22 activity $(4.3 \pm 0.6)$, pain $(4.2 \pm 0.7)$, and total $(4.2 \pm 0.4)$ scores than those in the Reop 30 days -1 Year Group (3.2 \pm 1.2 , p $=0.0477 ; 3.0 \pm 1.2, \mathrm{p}=0.0483$; and $3.1 \pm 1.0, \mathrm{p}=0.0278$, respectively).

\section{Reoperation Impact on Clinical Outcomes: Patients With at Least 2 Years of Follow-Up}

Reoperation Within 1 Year Versus No Reoperation. There were no significant differences between the Reop Within 1 Year and No Reop groups for any of the HRQOL outcomes at 1- or 2-year time points $(\mathrm{p}>0.05)$.

Reoperation After 1 Year Versus No Reoperation. There were no significant differences between the Reop After 1 Year and No Reop groups for any 1-year HRQOL outcomes $(p>0.05)$. Patients who underwent reoperation more than 1 year after the index surgery had significantly lower 2-year SF-36 physical component summary scores $(35.6 \pm 10.4)$ and SRS-22 pain scores $(3.0 \pm 1.0)$ than those who did not have reoperation $(42.5 \pm 11.6, \mathrm{p}$ $=0.0311 ; 3.5 \pm 1.1, \mathrm{p}=0.0429$, respectively). In addition, patients who did not undergo reoperation had a significantly larger increase in the 2-year PCS (compared with preoperative scores) than the Reop After 1 Year Group (8.7 \pm 10.9 vs. $1.8 \pm 8.9, \mathrm{p}=0.0242)$.

Reoperation Within 30 Days Versus Reoperation 30 Days-1 Year After the Index Surgery. There were no significant differences between the Reop Within 30 Days and the Reop 30 Days-1 Year groups for any 1-year HRQOL outcomes $(p>0.05)$. At the 2-year follow-up, those who underwent reoperation less than 30 days after the index procedure had significantly better SRS activity 


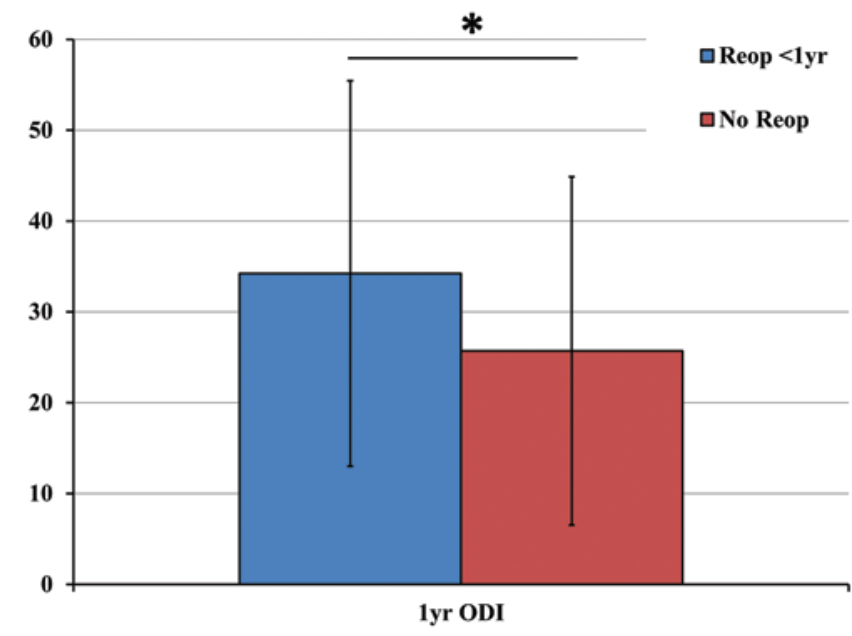

FIG. 1. Mean total ODI score for patients who underwent reoperation within 1 year compared with score for those who did not.

$(4.3 \pm 0.6)$, pain $(4.2 \pm 0.7)$, and total $(4.2 \pm 0.4)$ scores than those who had reoperation more than 30 days and less than 1 year after the index procedure $(3.2 \pm 1.2, \mathrm{p}=$ $0.0477,3.0 \pm 1.2, \mathrm{p}=0.0483$, and $3.1 \pm 1.0, \mathrm{p}=0.0278$, respectively).

\section{Discussion}

Analysis of a large multicenter adult spinal deformity database shows an overall reoperation rate of $17 \%$ at a mean follow-up of 1.6 years following the initial procedure. This is comparable to previous studies that have reported rates ranging from $10 \%$ to $25 \%$. $4,13,17$

This study has shown that age may not have a sig- nificant effect on the reoperation rate, with the exception of patients undergoing reoperation within 1 year of index surgery. Patients who underwent reoperation within 30 days of index surgery were significantly younger than those who underwent reoperation within 1 year but more than 30 days after the index procedure.

Of the indications requiring reoperation, the most common included instrumentation complications and radiographic failure. This reinforces the importance of preoperative planning, intraoperative imaging, and surgical technique. Lastly, the results show that reoperation within 1 year does have an effect on HRQOL at 1 year for the minimum 1-year follow-up cohort. The patients who had reoperation had significantly worse outcomes in many areas of HRQOL. This result suggests that a second surgery within 1 year of the index surgery can have an adverse effect on the patient, including a significant impact on pain, disability and SRS-22 subscores. The poorer HRQOL also further emphasizes the importance of critical preoperative planning and careful surgical technique to attempt to minimize the potential need for short-term reoperation. Despite the difference in 1-year HRQOL scores, at 2 years the scores normalized and there was no significant difference in any of them between the 2 groups. The minimum 2-year follow-up cohort did not show any significant difference in HRQOL between patients who underwent reoperation within 1 year of the index procedure and those who did not undergo reoperation. This may be a result of patients being lost to followup, as there were 31 patients who had reoperation within 1 year in the 1-year minimum follow-up cohort and only 16 in the 2-year minimum follow-up cohort. This could alter the results away from any effect on 1-year outcomes as about half of those patients were not seen at 2 years.

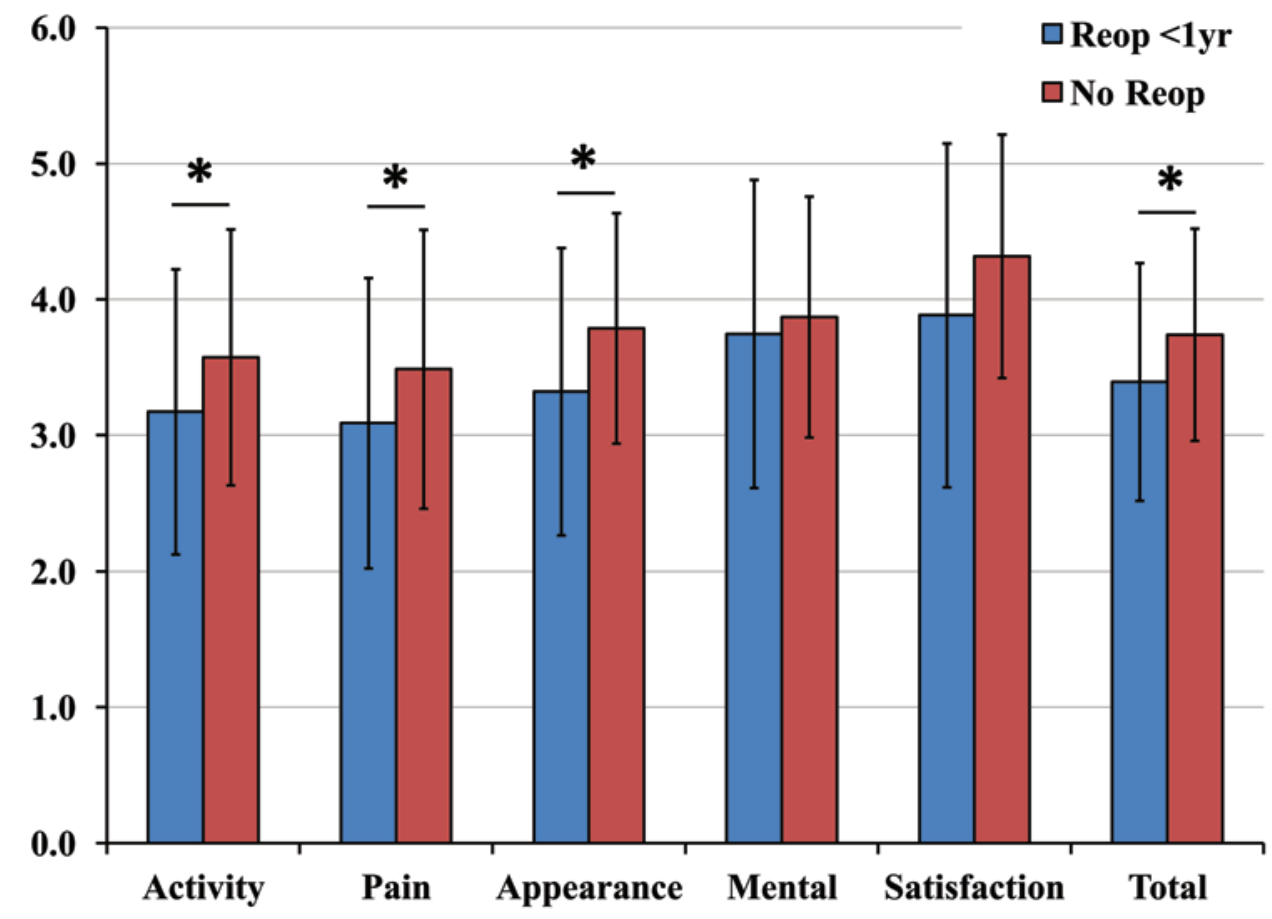

FIG. 2. Graphical display of the SRS-22 subscore analysis comparing scores for patients who underwent reoperation within 1 year of the index procedure and those who did not. 
The 2-year minimum follow-up cohort results should not discount the results from the 1-year minimum follow-up cohort because those patient who had reoperations within 1 year in the 1-year minimum follow-up cohort were followed up for at least a year and did have an effect on 1 -year outcomes. Similarly the patients who had reoperation more than 1 year after the index surgery in the 2 -year minimum follow-up cohort had a significant negative effect on HRQOL results at 2 years.

Of 53 patients treated with 3-column osteotomy, 19\% required one or more reoperations. Six of these patients underwent reoperation within 1 year of the index surgery, 4 underwent reoperation more than 1 year after the index surgery, and 2 of the 3 patients in this study requiring a second reoperation had undergone 3-column osteotomies. Given the complex nature of 3-column osteotomies, it is not surprising that the most common indications for reoperation were implant failure and neurological compromise, both of which are likely to occur early after surgery.

Even though pseudarthrosis has been reported as one of the most commonly documented reasons for reoperation, $, 6,10,13,16-18$ it is generally evaluated later than of 1 year after the index surgery. This study has focused on reoperation within 1 year and its effect on HRQOL. The need for reoperation may possibly be minimized by careful planning and execution of a number of factors, including spinal alignment, termination of fixation, and most importantly, precautions that could be taken to avoid malposition or instrumentation (rod) failure, such as usage of additional satellite rods across the 3-column osteotomy.

\section{Conclusions}

Adult deformity spine surgery remains technically challenging, with high rates of complications that may lead to reoperations. An analysis of data from a large multicenter adult spinal deformity database showed an overall $17 \%$ reoperation rate, with a $19 \%$ reoperation rate for patients treated with 3-column osteotomy and a $16 \%$ reoperation rate for patients not treated with 3-column osteotomy. The most common indications for reoperation included instrumentation complications and radiographic failure. Undergoing a reoperation within 1 year of index surgery significantly adversely affected HRQOL measures at 1-year follow-up. The need for reoperation may be minimized by carefully considering spinal alignment, termination of fixation, and type of surgical procedure (presence of osteotomy). Precautions should be taken to avoid malposition or instrumentation (rod) failure.

\section{Disclosure}

Dr. Shaffrey reports a patent holder relationship with Medtronic and Biomet; a consultant relationship with Medtronic, Biomet, NuVasive, and Globus; and receiving clinical or research support for this study from DePuy. Dr. Ames reports a consultant relationship with DePuy, Medtronic, and Stryker; a patent holder relationship with Fish \& Richardson, P.C.; direct stock ownership in Trans1, Visualase, and Doctors Research Group; and receiving royalties from Lanx and Aesculap. Dr. Smith reports a consultant relationship with Biomet and Medtronic and receiving clinical or research sup- port for this study from DePuy. Dr. Hart reports receiving royalties from and/or being on the speakers' bureau of DePuy, Seaspine, Kyphon, and Synthes; a consultant relationship with DePuy, Eli Lilly, and Medtronic; direct stock ownership in SpineConnect; receiving support for non-study related clinical or research efforts from DePuy, Medtronic, OREF, and Synthes; and holding a patent with OHSU. Dr. Lafage reports a consultant relationship with Medtronic and direct stock ownership in Nemaris Inc. Dr. Burton reports a consultant relationship with and receiving royalties from DePuy Spine, and board membership on University of Kansas Physicians, Inc. and the ISSG. Dr. Bess reports a consultant relationship with Medtronic, DePuy Spine, Alphatec, and Allosource and receiving clinical or research support for this study from DePuy Spine. Dr. Mundis reports a consultant relationship with NuVasive and $\mathrm{K} 2 \mathrm{M}$ and receiving clinical or research support for this study from NuVasive, K2M, and OREF. Dr. Klineberg reports a consultant relationship with DePuy, Synthes, Alphatec, and Stryker; and receiving support for non-study related clinical or research efforts from AO Spine Research, OREF, and Synthes. Dr. O'Brien reports a consultant relationship with DePuy and NuVasive and receiving clinical or research support for the study described from DePuy and NuVasive.

Author contributions to the study and manuscript preparation include the following. Conception and design: Ames. Acquisition of data: Scheer. Analysis and interpretation of data: Scheer, Tang, Smith. Drafting the article: Scheer, Tang, Smith, Shaffrey. Critically revising the article: all authors. Reviewed submitted version of manuscript: all authors. Approved the final version of the manuscript on behalf of all authors: Scheer. Statistical analysis: Scheer. Administrative/technical/material support: Ames. Study supervision: Ames.

\section{References}

1. Acosta FL Jr, McClendon J Jr, O'Shaughnessy BA, Koller H, Neal CJ, Meier O, et al: Morbidity and mortality after spinal deformity surgery in patients 75 years and older: complications and predictive factors. Clinical article. J Neurosurg Spine 15: 667-674, 2011

2. Asher M, Min Lai S, Burton D, Manna B: The reliability and concurrent validity of the scoliosis research society-22 patient questionnaire for idiopathic scoliosis. Spine (Phila Pa 1976) 28:63-69, 2003

3. Auerbach JD, Lenke LG, Bridwell KH, Sehn JK, Milby AH, Bumpass D, et al: Major complications and comparison between 3-column osteotomy techniques in 105 consecutive spinal deformity procedures. Spine (Phila Pa 1976) 37:11981210,2012

4. Cook S, Asher M, Lai SM, Shobe J: Reoperation after primary posterior instrumentation and fusion for idiopathic scoliosis. Toward defining late operative site pain of unknown cause. Spine (Phila Pa 1976) 25:463-468, 2000

5. Drazin D, Shirzadi A, Rosner J, Eboli P, Safee M, Baron EM, et al: Complications and outcomes after spinal deformity surgery in the elderly: review of the existing literature and future directions. Neurosurg Focus 31(4):E3, 2011

6. Emami A, Deviren V, Berven S, Smith JA, Hu SS, Bradford DS: Outcome and complications of long fusions to the sacrum in adult spine deformity: luque-galveston, combined iliac and sacral screws, and sacral fixation. Spine (Phila Pa 1976) 27: 776-786, 2002

7. Hu RW, Jaglal S, Axcell T, Anderson G: A population-based study of reoperations after back surgery. Spine (Phila Pa 1976) 22:2265-2271, 1997

8. Kim YJ, Bridwell KH, Lenke LG, Glattes CR, Rhim S, Cheh G: Proximal junctional kyphosis in adult spinal deformity after segmental posterior spinal instrumentation and fusion: minimum five-year follow-up. Spine (Phila Pa 1976) 33: 2179-2184, 2008

9. Kim YJ, Bridwell KH, Lenke LG, Rhim S, Cheh G: Pseudarthrosis in long adult spinal deformity instrumentation and fu- 
sion to the sacrum: prevalence and risk factor analysis of 144 cases. Spine (Phila Pa 1976) 31:2329-2336, 2006

10. Kim YJ, Bridwell KH, Lenke LG, Rinella AS, Edwards C II Pseudarthrosis in primary fusions for adult idiopathic scoliosis: incidence, risk factors, and outcome analysis. Spine (Phila Pa 1976) 30:468-474, 2005

11. Lapp MA, Bridwell KH, Lenke LG, Daniel Riew K, Linville DA, Eck KR, et al: Long-term complications in adult spinal deformity patients having combined surgery a comparison of primary to revision patients. Spine (Phila Pa 1976) 26:973983, 2001

12. Linville DA, Bridwell KH, Lenke LG, Vedantam R, Leicht P: Complications in the adult spinal deformity patient having combined surgery. Does revision increase the risk? Spine (Phila Pa 1976) 24:355-363, 1999

13. Luhmann SJ, Lenke LG, Bridwell KH, Schootman M: Revision surgery after primary spine fusion for idiopathic scoliosis. Spine (Phila Pa 1976) 34:2191-2197, 2009

14. Malter AD, McNeney B, Loeser JD, Deyo RA: 5-year reoperation rates after different types of lumbar spine surgery. Spine (Phila Pa 1976) 23:814-820, 1998

15. Martin BI, Mirza SK, Comstock BA, Gray DT, Kreuter W, Deyo RA: Reoperation rates following lumbar spine surgery and the influence of spinal fusion procedures. Spine (Phila Pa 1976) 32:382-387, 2007

16. Mok JM, Cloyd JM, Bradford DS, Hu SS, Deviren V, Smith JA, et al: Reoperation after primary fusion for adult spinal deformity: rate, reason, and timing. Spine (Phila Pa 1976) 34: 832-839, 2009
17. Pichelmann MA, Lenke LG, Bridwell KH, Good CR, O'Leary PT, Sides BA: Revision rates following primary adult spinal deformity surgery: six hundred forty-three consecutive patients followed-up to twenty-two years postoperative. Spine (Phila Pa 1976) 35:219-226, 2010

18. Ramo B, Richards BS: Repeat surgical interventions following "definitive" instrumentation and fusion for idiopathic scoliosis: five-year update on a previously published cohort. Spine (Phila Pa 1976) 37:1211-1217, 2012

19. Rinella A, Bridwell K, Kim Y, Rudzki J, Edwards C, Roh M, et al: Late complications of adult idiopathic scoliosis primary fusions to L4 and above: the effect of age and distal fusion level. Spine (Phila Pa 1976) 29:318-325, 2004

20. Smith JS, Shaffrey CI, Glassman SD, Berven SH, Schwab FJ, Hamill CL, et al: Risk-benefit assessment of surgery for adult scoliosis: an analysis based on patient age. Spine (Phila Pa 1976) 36:817-824, 2011

Manuscript submitted September 23, 2012.

Accepted July 15, 2013.

Please include this information when citing this paper: published online August 23, 2013; DOI: 10.3171/2013.7.SPINE12901.

Address correspondence to: Justin K. Scheer, B.S., UC San Diego School of Medicine, 9500 Gilman Dr., La Jolla, CA 92093. email: jscheer@ucsd.edu. 